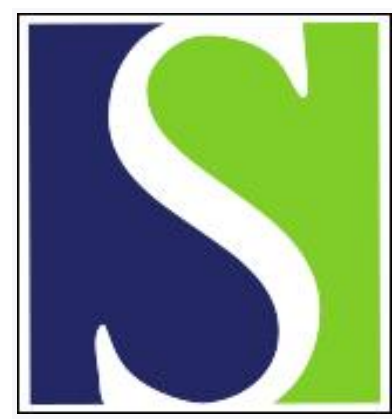

Scand J Work Environ Health 2020;46(4):410-416

https://doi.org/10.5271/sjweh.3888

Published online: 20 Feb 2020, Issue date: 01 Jul 2020

Neurodevelopmental disorders among young adults and the risk of sickness absence and disability pension: a nationwide register linkage study

by Virtanen M, Lallukka T, Kivimäki M, Alexanderson K, Ervasti J, Mittendorfer-Rutz E

This register-based study of the entire Swedish population found that attention-deficit/hyperactivity disorder, autism spectrum disorder, and learning disabilities had major effects on the lives of young adults in terms of sickness absence and disability pension. For the first time, we also examined the contribution of socio-demographic factors and comorbid mental disorders to the examined outcomes.

Affiliation: Karolinska Institutet, Division of Insurance Medicine, SE-171 77 Stockholm, Sweden. marianna.virtanen@uef.fi

Refers to the following text of the Journal: 2020;46(1):50-59

Key terms: ADHD; attention-deficit hyperactivity disorder; autism; disability pension; learning disability; neurodevelopmental disorder; register study; sick leave; sickness absence; young adult

This article in PubMed: www.ncbi.nlm.nih.gov/pubmed/32076730

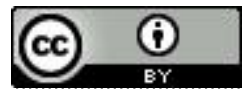




\title{
Neurodevelopmental disorders among young adults and the risk of sickness absence and disability pension: a nationwide register linkage study
}

\author{
by Marianna Virtanen, PhD, 1,2 Tea Lallukka, PhD, ${ }^{3,4}$ Mika Kivimäki, FMedSci, ${ }^{3,5}$ Kristina Alexanderson, PhD, ${ }^{1}$ Jenni Ervasti, \\ PhD, ${ }^{4}$ Ellenor Mittendorfer-Rutz, $P h D^{1}$
}

\begin{abstract}
Virtanen M, Lallukka T, Kivimäki M, Alexanderson K, Ervasti J, Mittendorfer-Rutz E. Neurodevelopmental disorders among young adults and the risk of sickness absence and disability pension: a nationwide register linkage study. Scand J Work Environ Health. 2020;46(4):410-416. doi:10.5271/sjweh.3888
\end{abstract}

\begin{abstract}
Objectives Attention-deficit/hyperactivity disorder (ADHD), autism spectrum disorders (ASD) and learning disabilities (LD) have an early onset and often persist into adulthood, although their relative contribution to incapacity for work is unclear. We examined this issue among young adults with ADHD, ASD or LD taking into account socioeconomic factors and comorbid mental disorders.
\end{abstract}

Methods Recorded diagnoses between the ages of 10-35 years between 2001 and 2010 were derived from nationwide inpatient and specialized outpatient hospital registers in Sweden. We identified 15632 individuals with a main diagnosis of ADHD, 8238 with ASD, and 1038 with LD, and the matched control group without recorded mental disorders $(\mathrm{N}=124$ 536). The outcome was the number of register-based sickness absence and work disability pension (SA-DP) days during a maximum of three years follow-up.

Results Among men, the rate ratio (RR) of SA-DP was 11.17 [95\% confidence interval (CI) 9.89-12.60] for ADHD, 35.59 (95\% CI 30.30-41.81) for ASD, and 9.20 (95\% CI 5.76-14.70) for LD, in comparison to those in the reference group. The corresponding risks among women were RR 12.05 (95\% CI 10.30-14.09) for ADHD, RR 28.36 (95\% CI 22.96-35.02) for ASD, and RR 9.60 (95\% CI 5.83-15.81) for LD. The findings were, to a large extent, similar when individuals on DP at baseline were excluded. Comorbid mental disorders further increased the risk of SA-DP. Educational differences were smaller among the patients than in the reference group.

Conclusions Early-onset neurodevelopmental disorders, particularly with comorbidity, have a far-reaching impact on adult life in terms of SA and DP.

Key terms ADHD; attention-deficit hyperactivity disorder; autism; learning disability; register study; sick leave.

Attention-deficit/hyperactivity disorder (ADHD), autism spectrum disorders (ASD), and learning disabilities (LD) usually begin in childhood and may significantly impact the future lives of affected children. These disorders often persist into adulthood, may increase the risk of other mental disorders, and are associated with failure in developmental tasks such as gaining education and employment (1-10). The prevalence of ADHD, ASD, and LD among adults has been estimated to be around 4\% (11), 1\% (12), and $1-2.5 \%$ (13), respectively, although exact estimates for adults are seldom available. Economic costs in terms of special educational needs, healthcare services, and productivity loss in the labor market are substantial (1, 10,14-17). Comorbid diseases are believed to be major drivers of healthcare utilization and cost (15-17).

The effects of early-onset neuropsychiatric and behavioral disorders may be particularly detrimental during young adulthood, since this period includes important developmental tasks, such as completing an education and entering into the labor market. To date, most studies have focused on ADHD, with the outcome usually being general 'productivity loss' measured as

1 Department of Clinical Neuroscience, Division of Insurance Medicine, Karolinska Institutet, Stockholm, Sweden.

2 School of Educational Sciences and Psychology, University of Eastern Finland, Joensuu, Finland.

3 Clinicum, Faculty of Medicine, University of Helsinki, Finland.

4 Finnish Institute of Occupational Health, Helsinki, Finland.

5 Department of Epidemiology and Public Health, University College London, UK.

Correspondence to: Marianna Virtanen, Karolinska Institutet, Division of Insurance Medicine, SE-171 77 Stockholm, Sweden. [E-mail: marianna.virtanen@uef.fi] 
costs $(1,17)$ or occupational injuries $(18)$. Two studies reported increased sickness absence (SA) days, assessed by self-reported days during the past month $(15,19)$. More short-term disability days but no difference between the likelihood of SA among workers with and without ADHD was reported in one study (16). However, these three studies did not examine other disorders than $\operatorname{ADHD}(15,16,19)$ or relied on self-reported outcome data $(15,19)$. We are not aware of any studies that have compared ADHD, ASD, and LD or examined whether socioeconomic factors and comorbid conditions are associated with the incapacity for work outcomes in these disorders.

Using register data covering the whole population of Sweden, we examined the longitudinal associations of ADHD, ASD, and LD with SA and disability pension (DP) among young adults, in comparison to those in a matched reference group without recorded mental disorders, and assessed the contribution of socioeconomic characteristics and comorbid mental disorders.

\section{Methods}

\section{Participants and procedure}

The study population was a register-based cohort derived from the whole population's register in Sweden, linked to in- and specialized outpatient registers between 2001 and 2010 (figure 1). The Regional Ethical Review Board, Stockholm, Sweden, approved the project. Statistics Sweden's Longitudinal Integration Database for
Health Insurance and Labor Market Studies (LISA) was used to obtain information on sex, age, education, birth country, and type of living area. The National Board of Health and Welfare provided data from the patient register (diagnosis-specific data on inpatient hospitalizations and specialized outpatient care), coded according to the International Classification of Diseases (ICD-10) and date of death. The National Social Insurance Agency provided information on annual days of SA-DP (the MIDAS register).

In Sweden, all individuals aged $\geq 16$ years are entitled to SA benefits if they have income from work or unemployment benefits and have work incapacity due to disease or injury. All people aged 19-64, including those with no previous income, can be granted DP if their work capacity is long-term or permanently reduced due to disease or injury. For people aged $\geq 30$ years, work incapacity has to be permanent before DP can be granted. Between ages 19-29, work incapacity needs to last for at least one year and can be granted to those still in school.

The individuals with neurodevelopmental disorders were those who had been 10-35 years old when their ADHD (International Classification of Diseases, ICD10 code F90), ASD (F84.0, F84.1, F84.3, F84.5, F84.8, F84.9), or LD (F81) was recorded as the main diagnosis in the specialized in- or outpatient healthcare between 2001-2010. An additional inclusion criterion was living in Sweden five years prior to the treatment episode of specialized healthcare. Of the 24908 individuals with a recorded diagnosis of ADHD, ASD, or $\mathrm{LD}, 65 \%$ were aged 10-18 at the time of their diagnosis in specialized healthcare, whereas $35 \%$ were aged $19-35$ years.

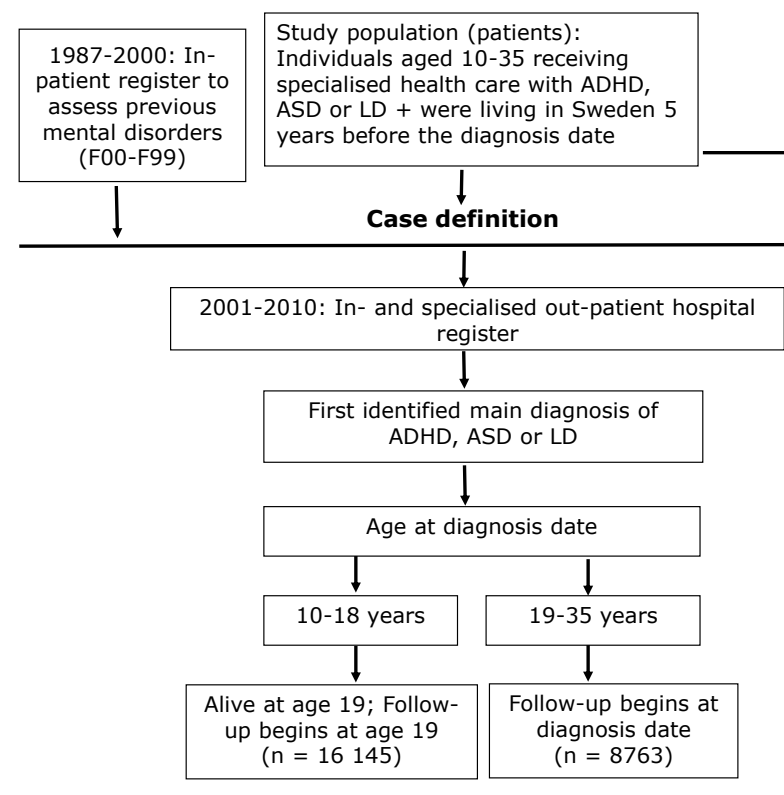

Follow-up: from the diagnosis date/age 19 until the end of 2013: max 3-year data on sickness absence and disability pension
Age-, sex-, living area and birth country matched referents ( 5 for each case at cohort entry date (being 10-35 years 2001-2010): with no recorded mental disorders (F00-F99) in the patient registers 1987-2013; living in Sweden 5 years before cohort entry date $(n=124536)$

Socio-demographic factors at the beginning of follow-up: from the year before the index diagnosis / year before age 19

Hospital records of co-morbid mental disorders before or at the time of the main diagnosis of ADHD, ASD or LD

Figure 1. Study population. [ADHD=attention-deficit/hyperactivity disorder; $A S D=$ autism spectrum disorders; $L D=$ learning disabilities.] 
We randomly selected five matched reference individuals $(\mathrm{N}=124$ 536) to each $\mathrm{ADHD}, \mathrm{ASD}$, and $\mathrm{LD}$ patient and matched them according to age, sex, type of living area, and birth country. The reference group had no indication of mental disorder (ICD-10 code F00-F99) in any of the hospital records. The individuals in the reference group had been living in Sweden five years prior to the cohort entry date of the patient with whom they were matched. A maximum of three years followup of annual net days of SA-DP began from the date of diagnosis, except when age at diagnosis was $<19$ years, in which case the follow-up began at the age of 19 (the eligibility age for DP in Sweden). The mean follow-up was 2.63 [standard deviation (SD) 0.69 ] years. The duration of follow-up depended on the year of entry into the cohort, death, and emigration. A total of 2593 individuals died or emigrated from Sweden during follow-up.

Covariates included sex, age, educational level (low: $0-9$, intermediate: $10-12$, high: $\geq 13$ years), birth country (Sweden, other), type of living area (large city, medium sized town, small town/village), and comorbid mental disorders before or at the time of the main diagnosis, also derived from inpatient and specialized outpatient registers. Comorbidity also included mental retardation (ICD-10 codes F70-F73, F78, F79) (20). We used the MIDAS work DP register to identify those who were on work DP at the beginning of follow-up.

\section{Statistical analysis}

Descriptive statistics (N, \%) for each diagnostic group and the reference group were calculated for covariates.
We calculated annual and 3-year net number of SA-DP days for each person (based on the sum of SA-DP). We applied negative binomial regression procedure to produce rate ratios (RR) and their $95 \%$ confidence intervals (CI) to estimate the difference in SA-DP between individuals with $\mathrm{ADHD}, \mathrm{ASD}$, or $\mathrm{LD}$, and the reference group. The offset variable was logarithmically transformed duration of follow-up (maximum three years) calculated for all individuals. Models were adjusted for socioeconomic factors and the calendar year when follow-up began. The analyses were repeated among a sub-group of individuals who were not on DP at the beginning of follow-up. Further analyses were carried out separately among cases and the control group to examine the association between socioeconomic factors and SA-DP, and among cases, the association between comorbid mental disorders and SA-DP. We used SAS statistical software (9.4) (SAS Institute, Inc, Cary, NC, USA).

\section{Results}

Table 1 presents the descriptive characteristics of individuals with $\mathrm{ADHD}, \mathrm{ASD}$ and $\mathrm{LD}$, and the reference group without recorded mental disorder. The largest diagnostic group was ADHD ( $\mathrm{N}=15$ 632), followed by ASD $(\mathrm{N}=8238)$, and LD $(\mathrm{N}=1038)$. Men and those with low education were overrepresented in all the diagnostic groups. The highest prevalence of comorbid mental disorders was found among individuals with ASD (48\%) and the majority of individuals with ASD $(63 \%)$ were

Table 1. Characteristics at the beginning of follow-up of individuals with attention-deficit hyperactivity disorder (ADHD), autism spectrum disorder (ASD) and learning disabilities (LD), and a matched reference group without recorded mental disorders. [NA=not applicable; $S D=$ standard deviation.]

\begin{tabular}{|c|c|c|c|c|c|c|c|c|}
\hline & \multicolumn{2}{|c|}{ Reference group ( $\mathrm{N}=124$ 536) } & \multicolumn{2}{|c|}{ ADHD (N=15 632) } & \multicolumn{2}{|c|}{ ASD (N=8238) } & \multicolumn{2}{|c|}{ LD ( $N=1038)$} \\
\hline & Mean (SD) & $\mathrm{N}(\%)$ & Mean (SD) & $\mathrm{N}(\%)$ & Mean (SD) & $\mathrm{N}(\%)$ & Mean (SD) & $\mathrm{N}(\%)$ \\
\hline Age at beginning of follow-up (reference) & $21.2(4.2)$ & & $21.2(4.2)$ & & $21.3(4.2)$ & & $20.0(3.0)$ & \\
\hline \multicolumn{9}{|l|}{ Sex } \\
\hline Men & & $86223(69)$ & & $10862(69)$ & & $5755(70)$ & & $628(61)$ \\
\hline Women & & $38313(31)$ & & 4770 (31) & & $2483(30)$ & & $410(40)$ \\
\hline \multicolumn{9}{|l|}{ Type of living area } \\
\hline Large city & & $41619(33)$ & & $5244(34)$ & & $2793(34)$ & & $287(28)$ \\
\hline Medium-sized town & & $45565(37)$ & & $5778(37)$ & & $2910(35)$ & & $425(41)$ \\
\hline Small town/village & & $37352(30)$ & & $4610(29)$ & & $2535(31)$ & & $326(31)$ \\
\hline \multicolumn{9}{|l|}{ Birth country } \\
\hline Sweden & & $116239(93)$ & & $14586(93)$ & & 7701 (93) & & $961(93)$ \\
\hline Other & & $8297(7)$ & & $1046(7)$ & & $537(7)$ & & $77(7)$ \\
\hline \multicolumn{9}{|l|}{ Educational level } \\
\hline Low & & $21741(17)$ & & $10065(64)$ & & $5530(67)$ & & $627(60)$ \\
\hline Medium & & $89684(72)$ & & $5041(32)$ & & $2328(28)$ & & $386(37)$ \\
\hline High & & $13111(11)$ & & $526(3)$ & & $380(5)$ & & $25(2)$ \\
\hline \multicolumn{9}{|l|}{ Comorbid mental disorder } \\
\hline No & & NA & & $9794(63)$ & & $4264(52)$ & & $753(73)$ \\
\hline Yes & & NA & & $5838(37)$ & & $3974(48)$ & & $285(27)$ \\
\hline \multicolumn{9}{|l|}{ Disability pension at beginning of follow-up } \\
\hline No & & 123227 (99) & & $12139(78)$ & & $3029(37)$ & & $825(79)$ \\
\hline Yes & & $1309(1)$ & & $3493(22)$ & & $5209(63)$ & & $213(21)$ \\
\hline
\end{tabular}


on DP already at the beginning of follow-up. The corresponding proportions for ADHD and LD were 22\% and $21 \%$, respectively.

Multivariable-adjusted RR for SA-DP days during the 3-year follow-up among men and women are presented in table 2. Compared to the reference group, ASD was associated with a 35.6-fold risk of SA-DP among men and a 28.4-fold risk among women. ADHD was associated with an 11.2-fold risk among men and a 12.1-fold risk among women whereas LD was associated with 9.2-fold risk among men and 9.6-fold risk among women. The findings were largely similar among a sub-group consisting men and women not on DP at the beginning of follow-up although the absolute rates were remarkably lower.

Among the patient groups, comorbid mental disorders further increased the SA-DP risk (table 3). The greatest RR (1.76) associated with comorbidity was found among those with ADHD. In ASD, the RR was 1.14 and in LD, the RR was 1.62 although not statistically significant in the latter group. Rather similar results were found among a sub-group of those not on DP at the beginning of follow-up.

The associations between baseline socioeconomic characteristics and SA-DP are presented in table 4. In all groups, women had higher risk than men. There was much greater differences between educational groups among the reference group without mental disorders (RR for low versus high education 18.40) than among the patients (RR 2.98). However, the estimate for low education in the reference group diluted to RR 4.02 when the individuals on DP at baseline were excluded from the analysis (the corresponding RR 2.10 among the patient group). Living in a small town or rural area was

Table 2. Rate ratio (RR) and 95\% confidence intervals (Cl) of sickness absence and disability pension days during follow-up among young adults diagnosed with attention-deficit/hyperactivity disorder (ADHD), autism spectrum disorder (ASD), or learning disabilities (LD) compared to matched reference group without recorded mental disorders.

\begin{tabular}{|c|c|c|c|c|}
\hline & \multicolumn{2}{|c|}{$\begin{array}{l}\text { Among the total } \\
\text { population } \\
(\mathrm{N}=149444)\end{array}$} & \multicolumn{2}{|c|}{$\begin{array}{l}\text { Among a sub-group not on } \\
\text { disability pension at beginning } \\
\text { of follow-up ( } \mathrm{N}=139220)\end{array}$} \\
\hline & $\begin{array}{l}\text { Unadjusted } \\
\text { rate per } \\
\text { person-year }\end{array}$ & $\begin{array}{l}\text { Adjusted RR } \\
(95 \% \mathrm{Cl})^{a}\end{array}$ & $\begin{array}{l}\text { Unadjusted } \\
\text { rate per } \\
\text { person-year }\end{array}$ & $\begin{array}{c}\text { Adjusted RR } \\
(95 \% \mathrm{Cl})^{\mathrm{a}}\end{array}$ \\
\hline \multicolumn{5}{|l|}{ Men } \\
\hline Reference & 5.1 & 1.00 & 2.2 & 1.00 \\
\hline ADHD & 88.1 & $11.17(9.89-12.60)$ & 29.3 & 9.97 (8.29-11.99) \\
\hline ASD & 247.9 & 35.59 (30.30-41.81) & 92.8 & $35.90(25.54-50.46)$ \\
\hline LD & 70.8 & $9.20(5.76-14.70)$ & 18.5 & $6.60(3.29-13.24)$ \\
\hline \multicolumn{5}{|l|}{ Women } \\
\hline Reference & 6.1 & 1.00 & 3.3 & 1.00 \\
\hline ADHD & 111.4 & $12.05(10.30-14.09)$ & 49.6 & $11.40(9.10-14.29)$ \\
\hline ASD & 263.2 & $28.36(22.96-35.02)$ & 98.3 & $25.03(16.22-38.65)$ \\
\hline $\mathrm{LD}$ & 78.4 & $9.60(5.83-15.81)$ & 19.2 & $6.01(2.99-12.07)$ \\
\hline
\end{tabular}

rather consistently associated with higher risk of SA-DP when compared to living in a big city. Non-Swedish birth country was associated with lower risk of disability among the reference group without mental disorders, while no differences were observed in the patient group.

Table 5 shows the annual difference between diagnostics groups and the reference group. Although the overall pattern was similar to the original analyses, the differences seemed to slightly increase among men and slightly decrease among women.

\section{Discussion}

In this register-based prospective study of the workingage population in Sweden, we found high risk in terms of SA-DP associated with ADHD, ASD and LD. Two in three of the patients were treated in the specialized healthcare during childhood or adolescence, which reflects the early onset of these disorders. Compared to the matched reference group without recorded mental disorders, ASD was associated with the greatest burden of SA-DP with a 36-fold risk among men and 28-fold risk among women. Of them, $63 \%$ were already on DP at the beginning of follow-up. The corresponding risks among men and women with ADHD were 11 and 12; and among men and women with LD, 9 and 10, respectively. However, comorbid mental disorders played a significant role, further increasing the SA-DP risk. Educational disparities in SA-DP were smaller in the patient than reference group.

Our findings suggest that ASD is associated with the greatest burden of SA-DP. ASD represent a category of disorders that are characterized by difficulties in social

Table 3. Rate ratio (RR) and 95\% confidence interval (Cl) of sickness absence and disability pension days during follow-up among young adults diagnosed with attention-deficit/hyperactivity disorder (ADHD), autism spectrum disorder (ASD), or learning disabilities (LD) by the presence of comorbid mental disorder

\begin{tabular}{|c|c|c|c|c|}
\hline \multirow[t]{2}{*}{$\begin{array}{l}\text { Diagnostic group } \\
\text { and comorbidity }\end{array}$} & \multicolumn{2}{|c|}{$\begin{array}{l}\text { Among the total } \\
\text { patient population } \\
(\mathrm{N}=24908)\end{array}$} & \multicolumn{2}{|c|}{$\begin{array}{l}\text { Among a sub-group not on } \\
\text { disability pension at begin- } \\
\text { ning of follow-up ( } N=15 \text { 993) }\end{array}$} \\
\hline & $\begin{array}{l}\text { Unadjusted } \\
\text { rate per } \\
\text { person-year }\end{array}$ & $\begin{array}{l}\text { Adjusted RR } \\
(95 \% \mathrm{Cl})^{\mathrm{a}}\end{array}$ & $\begin{array}{l}\text { Unadjusted } \\
\text { rate per } \\
\text { person-year }\end{array}$ & $\begin{array}{l}\text { Adjusted RR } \\
(95 \% \mathrm{Cl})^{\mathrm{a}}\end{array}$ \\
\hline \multicolumn{5}{|l|}{ ADHD } \\
\hline No comorbidity & 73.1 & 1.00 & 27.9 & 1.00 \\
\hline $\begin{array}{l}\text { Comorbidity } \\
\text { ASD }\end{array}$ & 132.3 & $1.76(1.57-1.97)$ & 50.3 & $1.60(1.33-1.92)$ \\
\hline No comorbidity & 233.8 & 1.00 & 81.4 & 1.00 \\
\hline $\begin{array}{l}\text { Comorbidity } \\
\text { LD }\end{array}$ & 272.7 & $1.14(1.06-1.23)$ & ) 111.4 & $1.41(1.09-1.82)$ \\
\hline No comorbidity & 61.1 & 1.00 & 15.2 & 1.00 \\
\hline Comorbidity & 107.5 & $1.62(0.90-2.92)$ & 29.9 & $1.49(0.42-5.26)$ \\
\hline
\end{tabular}

adjusted for age, sex, educational level, type of living area, birth country and year when follow-up began. 
reciprocity, communication and unusual or repetitive behavior. Among high-functioning people with ASD, difficulties in communication and social interaction rather than their actual work performance have been suggested to be the greatest obstacles to employment (4). Incapacity for work reflects a mismatch between job demands and an individual's capacity to respond to these demands due to a physical disease or mental disorder. However, people with ASD often have special, although narrow skills and high competence, and it may be possible to support their employment and capacity for work. They may need specific support at the workplace; eg, specific time devoted to communication and a structured, non-complex work environment (4).

Our findings correspond with previous studies that have shown associations between ADHD and self- reported one-month SA days $(15,19)$, and short-term disability days (16). ADHD is associated with symptoms that may substantially reduce work performance, such as abnormal attention, impulsiveness, hyperactivity or restlessness, disorganization, and time management and memory problems (14). People with ADHD may also have problems in motor coordination as well as in working memory, planning and anticipation, verbal fluency, effort allocation, and self-regulation of emotional arousal. However, they often have a high level of energy and enthusiasm, which may be a great advantage in favorable circumstances. Therefore, it has been recommended that regarding employees with ADHD, special attention should be paid to calming the work environment (eg, by avoiding noise and open offices). They should be allowed to move during the workday

Table 4. Baseline characteristics associated with the rate ratio (RR) and $95 \%$ confidence interval (Cl) of sickness absence and disability pension days during follow-up among young adults with attention-deficit/hyperactivity disorder (ADHD), autism spectrum disorder (ASD), or learning disabilities (LD) and among a reference group without recorded mental disorders.

\begin{tabular}{|c|c|c|c|c|}
\hline \multirow[t]{3}{*}{ Characteristics at baseline } & \multicolumn{2}{|c|}{$\begin{array}{l}\text { Among the total population } \\
\qquad(\mathrm{N}=149444)\end{array}$} & \multicolumn{2}{|c|}{$\begin{array}{l}\text { Among a sub-group not on disability pension } \\
\text { at beginning of follow-up }(\mathrm{N}=139220)\end{array}$} \\
\hline & $\begin{array}{l}\text { Reference group without mental } \\
\text { disorders }(\mathrm{N}=124536)\end{array}$ & $\begin{array}{l}\text { Individuals with ADHD, } \\
\text { ASD or LD }(N=24908)\end{array}$ & $\begin{array}{c}\text { Reference group without } \\
\text { mental disorders ( } \mathrm{N}=123227) \\
\end{array}$ & $\begin{array}{l}\text { Individuals with ADHD, } \\
\text { ASD or } L D(N=15993)\end{array}$ \\
\hline & $\operatorname{RR}(95 \% \mathrm{Cl})^{\text {a }}$ & $\operatorname{RR}(95 \% \mathrm{CI})^{\text {a }}$ & $\operatorname{RR}(95 \% \mathrm{Cl})^{\text {a }}$ & $\operatorname{RR}(95 \% \mathrm{Cl})^{\text {a }}$ \\
\hline \multicolumn{5}{|l|}{ Age at cohort entry (years) } \\
\hline$\leq 15$ & 1.00 & 1.00 & 1.00 & 1.00 \\
\hline$>15$ & $1.01(0.91-1.13)$ & $1.03(0.95-1.13)$ & $0.98(0.87-1.10)$ & $1.46(1.23-1.74)$ \\
\hline \multicolumn{5}{|l|}{ Sex } \\
\hline Men & 1.00 & 1.00 & 1.00 & 1.00 \\
\hline Women & $1.35(1.22-1.49)$ & $1.16(1.08-1.25)$ & $1.36(1.23-1.52)$ & $1.31(1.12-1.53)$ \\
\hline \multicolumn{5}{|l|}{ Educational level } \\
\hline High & 1.00 & 1.00 & 1.00 & 1.00 \\
\hline Intermediate & $2.35(1.98-2.79)$ & $1.20(0.98-1.47)$ & $2.16(1.80-2.59)$ & $1.17(0.81-1.68)$ \\
\hline Low & $18.40(15.04-22.52)$ & $2.98(2.43-3.65)$ & $4.02(3.25-4.98)$ & $2.10(1.44-3.07)$ \\
\hline \multicolumn{5}{|l|}{ Type of living area } \\
\hline Large city & 1.00 & 1.00 & 1.00 & 1.00 \\
\hline Medium-sized town & $1.22(1.10-1.36)$ & $1.03(0.94-1.12)$ & $1.10(0.98-1.23)$ & $1.15(0.97-1.36)$ \\
\hline Small town/village & $1.38(1.23-1.54)$ & $1.08(0.99-1.18)$ & $1.31(1.17-1.48)$ & $1.22(1.02-1.46)$ \\
\hline \multicolumn{5}{|l|}{ Birth country } \\
\hline Sweden & 1.00 & 1.00 & 1.00 & 1.00 \\
\hline Other & $0.73(0.61-0.88)$ & $1.03(0.89-1.18)$ & $0.70(0.58-0.85)$ & $0.96(0.71-1.29)$ \\
\hline
\end{tabular}

${ }^{a}$ Adjusted for age, sex, educational level, type of living area, birth country, and the year when follow-up began.

Table 5. Adjusted rate ratio (RR) and $95 \%$ confidence intervals $(\mathrm{Cl})$ of the annual sickness absence and disability pension days among young adults diagnosed with attention-deficit/hyperactivity disorder (ADHD), autism spectrum disorder (ASD) or learning disabilities (LD) compared to a matched reference group without recorded mental disorders. The analyses are based on persons with full year follow-up periods.

\begin{tabular}{|c|c|c|c|c|}
\hline & Reference & ADHD & ASD & LD \\
\hline & group & $\operatorname{RR}(95 \% \mathrm{Cl})^{\text {a }}$ & $\operatorname{RR}(95 \% \mathrm{Cl})^{\text {a }}$ & $\operatorname{RR}(95 \% \mathrm{CI})^{\text {a }}$ \\
\hline \multicolumn{5}{|l|}{ Men } \\
\hline Year 1 & 1.00 & $11.91(10.31-13.75)$ & $36.21(30.05-43.65)$ & $10.10(5.78-17.64)$ \\
\hline Year 2 & 1.00 & $12.30(10.61-14.25)$ & 39.24 (32.41-47.51) & $10.65(6.02-18.84)$ \\
\hline Year 3 & 1.00 & $12.88(11.00-15.07)$ & $39.96(32.75-48.74)$ & $11.97(6.48-22.12)$ \\
\hline \multicolumn{5}{|l|}{ Women } \\
\hline Year 1 & 1.00 & $13.58(11.18-16.48)$ & $31.41(24.26-40.67)$ & $11.21(5.94-21.18)$ \\
\hline Year 2 & 1.00 & $12.50(10.30-15.17)$ & $29.76(23.02-38.48)$ & $9.05(4.82-16.97)$ \\
\hline Year 3 & 1.00 & $12.04(9.83-14.76)$ & $28.79(22.07-37.58)$ & $8.77(4.45-17.32)$ \\
\hline
\end{tabular}

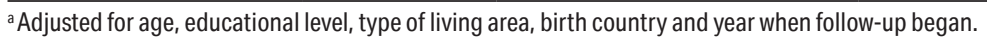


and receive help in organizing and keeping time schedules, and planning work tasks. They should be able to delegate work and receive support from supervisors and co-workers (14). Early ADHD has been found to be associated with later mental and substance use disorders, early pregnancy, school drop-out and criminality (5), which provides an idea of the potential mechanism linking early ADHD with future incapacity for work. In addition, behavior-based treatments may be effective, in addition to stimulant medication treatment (21).

LD refers to a heterogeneous group of neurodevelopmental disorders that influence the individual's ability to maintain, process or convey information to others effectively (22). This is often comorbid with other neurodevelopmental disorders such as ADHD (2). The literature on adult outcomes of LD is limited, and focuses mainly on educational and employment outcomes, showing mixed findings. The authors of a recent systematic review (2) concluded that comorbid mental disorders are the primary drivers of unfavorable outcomes in LD and a source of heterogeneity among previous studies. Our findings give some support to this hypothesis although the associations of comorbidity were not statistically significant.

Of the socioeconomic factors, educational disparities in SA-DP were smaller in the patient groups than the reference group without recorded mental disorders. It is possible that having a neurodevelopmental disorder per se is highly disabling and therefore less affected by social or environmental factors. There is some evidence from the US that neurodevelopmental disorders, such as ASD are underdiagnosed among socioeconomically disadvantaged groups due to worse resources and poorer access to health services compared to more affluent groups (23). If this was the case also in Sweden, proportionally more undiagnosed cases with socioeconomic disadvantage would have been in our reference group. These issues need to be investigated in detail in future studies. In general, the reference group consisted of young people who were not treated in specialized mental healthcare but may have had treatment contact in primary healthcare.

The specific strength of this study is its design based on nationwide registers that cover the entire Swedish population, and the high coverage and good validity of the Swedish health registers (24). The large database enabled us to investigate young adults with relatively rare neurodevelopmental disorders. The associations were quite similar in sub-group analyses excluding those with DP at baseline, although the absolute rates were lower, as expected.

However, the number of individuals with LD was lower than would be expected from prevalence statistics; the individuals with LD in our study were probably severe enough to be treated in a specialized healthcare setting. Furthermore, some individuals may have had their diagnosis before age 10 and not been in contact with specialized healthcare after that. However, as the registers cover both specialized outpatient clinics and inpatient healthcare, we do not consider this a major source of misclassification. Regarding comorbidity, some milder comorbid disorders, such as milder depressive disorders, may have had been misclassified because they are often treated in primary healthcare. The follow-up in our study was three years which reflects relatively short-term impacts of neuropsychiatric diseases in adult life. Work incapacity usually increases with age, thus future studies with longer follow-up may observe greater increases. The MIDAS register of SA-DP is valid in terms of high coverage and being based on a physician-assessed diagnosis of a disease and disability. One limitation is that our findings may be generalizable to cases treated in specialized healthcare and may not be generalizable to countries with very different social and healthcare systems. Finally, there are other risk and protective factors that were not assessed in the present study.

In conclusion, our findings suggest that ADHD, ASD, and LD have major effects on the lives of young adults in terms of incapacity for work. The greatest burden was found to be associated with ASD. Specific attention should be paid to co-occurring mental disorders that considerably impair the prognosis. Efforts to improve employment in these groups may include, for example, the individual placement and support (IPS) method which has been shown to be effective among young adults (25).

\section{Acknowledgements}

MK is supported by UK Medical Research Council (MR/RO24227/1), Academy of Finland (311492) and Helsinki Institute of Life Sciences. TL is supported by Academy of Finland $(287488,319200)$. The database was financially supported by the Swedish Research Council on Health, Working Life and Welfare (FORTE).

The authors declare no conflicts of interest.

\section{References}

1. Doshi JA, Hodgkins P, Kahle J, Sikirica V, Cangelosi MJ, Setyawan J, et al. Economic impact of childhood and adult attention-deficit/hyperactivity disorder in the United States. J Am Acad Child Adolesc Psychiatry 2012;51(10):990-1002 e2. https://doi.org/10.1016/j.jaac.2012.07.008.

2. Sharfi K, Rosenblum S. Activity and participation 
characteristics of adults with learning disabilities--a systematic review. PLoS One 2014;9(9):e106657. https://doi. org/10.1371/journal.pone.0106657.

3. Baxter AJ, Brugha TS, Erskine HE, Scheurer RW, Vos T, Scott JG. The epidemiology and global burden of autism spectrum disorders. Psychol Med 2015;45(3):601-13. https://doi. org/10.1017/S003329171400172X.

4. Scott M, Falkmer M, Girdler S, Falkmer T. Viewpoints on factors for successful employment for adults with autism spectrum disorder. PLoS One 2015;10(10):e0139281. https:// doi.org/10.1371/journal.pone.0139281.

5. Erskine HE, Norman RE, Ferrari AJ, Chan GC, Copeland WE, Whiteford HA, et al. Long-term outcomes of attention-deficit/ hyperactivity disorder and conduct disorder: A systematic review and meta-analysis. J Am Acad Child Adolesc Psychiatry 2016;55(10):841-50. https://doi.org/10.1016/j. jaac.2016.06.016

6. Hechtman L, Swanson JM, Sibley MH, Stehli A, Owens EB, Mitchell JT, et al. Functional adult outcomes 16 years after childhood diagnosis of attention-deficit/hyperactivity disorder: MTA results. J Am Acad Child Adolesc Psychiatry 2016;55(11):945-52. e2. https://doi.org/10.1016/j. jaac.2016.07.774.

7. Mohr-Jensen C, Steinhausen HC. A meta-analysis and systematic review of the risks associated with childhood attention-deficit hyperactivity disorder on long-term outcome of arrests, convictions, and incarcerations. Clin Psychol Rev 2016;48:32-42. https://doi.org/10.1016/j.cpr.2016.05.002.

8. Thapar A, Cooper M. Attention deficit hyperactivity disorder. Lancet 2016;387(10024):1240-50. https://doi.org/10.1016/ S0140-6736(15)00238-X.

9. Lord C, Elsabbagh M, Baird G, Veenstra-Vanderweele J. Autism spectrum disorder. Lancet. 2018. https://doi. org/10.1016/S0140-6736(18)31129-2.

10. Sayal K, Prasad V, Daley D, Ford T, Coghill D. ADHD in children and young people: prevalence, care pathways, and service provision. Lancet Psychiatry 2018;5(2):175-86. https://doi.org/10.1016/S2215-0366(17)30167-0.

11. Fayyad J, Sampson NA, Hwang I, Adamowski T, AguilarGaxiola S, Al-Hamzawi A, et al. The descriptive epidemiology of DSM-IV Adult ADHD in the World Health Organization World Mental Health Surveys. Atten Defic Hyperact Disord 2017;9(1):47-65. https://doi.org/10.1007/s12402-016-02083.

12. Lai MC, Lombardo MV, Baron-Cohen S. Autism. Lancet 2014;383(9920):896-910. https://doi.org/10.1016/S01406736(13)61539-1

13. Gillberg C, Soderstrom H. Learning disability. Lancet 2003;362(9386):811-21. https://doi.org/10.1016/S01406736(03)14275-4.

14. Adamou M, Arif M, Asherson P, Aw TC, Bolea B, Coghill D, et al. Occupational issues of adults with ADHD. BMC Psychiatry. 2013;13:59. https://doi.org/10.1186/1471-244X-13-59.

15. Kessler RC, Lane M, Stang PE, Van Brunt DL. The prevalence and workplace costs of adult attention deficit hyperactivity disorder in a large manufacturing firm. Psychol Med 2009;39(1):137-47.https://doi.org/10.1017/ S0033291708003309.

16. Hodgkins P, Montejano L, Sasane R, Huse D. Cost of illness and comorbidities in adults diagnosed with attention-deficit/ hyperactivity disorder: a retrospective analysis. Prim Care Companion CNS Disord 2011;13(2). https://doi.org/10.4088/ PCC.10m01030.

17. Libutzki B, Ludwig S, May M, Jacobsen RH, Reif A, Hartman CA. Direct medical costs of ADHD and its comorbid conditions on basis of a claims data analysis. Eur Psychiatry 2019;58:3844. https://doi.org/10.1016/j.eurpsy.2019.01.019.

18. Breslin FC, Pole JD. Work injury risk among young people with learning disabilities and attention-deficit/hyperactivity disorder in Canada. Am J Public Health 2009;99(8):1423-30. https://doi.org/10.2105/AJPH.2008.140855.

19. de Graaf R, Kessler RC, Fayyad J, ten Have M, Alonso $\mathrm{J}$, Angermeyer $\mathrm{M}$, et al. The prevalence and effects of adult attention-deficit/hyperactivity disorder (ADHD) on the performance of workers: results from the WHO World Mental Health Survey Initiative. Occup Environ Med 2008;65(12):835-42. https://doi.org/10.1136/ oem.2007.038448.

20. Hirvikoski T, Mittendorfer-Rutz E, Boman M, Larsson $\mathrm{H}$, Lichtenstein P, Bolte S. Premature mortality in autism spectrum disorder. Br J Psychiatry 2016;208(3):232-8. https:// doi.org/10.1192/bjp.bp.114.160192.

21. Fabiano GA, Pelham WE, Jr., Coles EK, Gnagy EM, ChronisTuscano A, O'Connor BC. A meta-analysis of behavioral treatments for attention-deficit/hyperactivity disorder. Clin Psychol Rev 2009;29(2):129-40. https://doi.org/10.1016/j. cpr.2008.11.001.

22. Kavale KA, Forness SR. What definitions of learning disability say and don't say: a critical analysis. J Learn Disabil 2000;33(3):239-56. https://doi. org/10.1177/002221940003300303.

23. Durkin MS, Maenner MJ, Baio J, Christensen D, Daniels J, Fitzgerald R, et al. Autism spectrum disorder among US children (2002-2010): Socioeconomic, racial, and ethnic disparities. Am J Public Health 2017;107(11):1818-26. https:// doi.org/10.2105/AJPH.2017.304032.

24. Ludvigsson JF, Andersson E, Ekbom A, Feychting M, Kim $\mathrm{JL}$, Reuterwall C, et al. External review and validation of the Swedish national inpatient register. BMC Public Health 2011;11:450. https://doi.org/10.1186/1471-2458-11-450.

25. Sveinsdottir V, Lie SA, Bond GR, Eriksen HR, Tveito TH, Grasdal AL, et al. Individual placement and support for young adults at risk of early work disability (the SEED trial). A randomized controlled trial. Scand J Work Environ Health 2020;46(1):50-9. https://doi.org/10.5271/sjweh.3837.

Received for publication: 19 June 2019 